\title{
A New Tracking Loop Scheme for High Dynamics Real-Time GNSS Receivers with Hardware Correlators
}

\author{
Pedro A. Roncagliolo and Javier G. García \\ Dto. Electrotecnia, Facultad Ingeniería, UNLP, 1900, La Plata, Argentina.
}

\section{BIOGRAPHY}

Pedro A. Roncagliolo received the Electronics Engineering and the Ph.D. in Engineering degrees from the National University of La Plata (UNLP), Argentina, in 2001 and 2009. Currently, he is a Professor in the UNLP involved in research and development of Global Navigation Satellite Systems (GNSS) receivers for aerospace applications. His research interests are in Statistical Signal Processing and Radio Frequency Electronics with applications to Wireless Communications and GNSS.

Javier G. García received the Electronics Engineering degree from the National University of La Plata (UNLP), Argentina, in 2003. He is currently a Professor in the UNLP, involved in research and development of Global Navigation Satellite Systems (GNSS) receivers for aerospace applications. His interests are in Statistical Signal Processing, Digital Communications, and Embedded Systems with applications to GNSS.

\section{ABSTRACT}

In this paper the implementation of one-delay carrier tracking loops in GNSS receivers that utilized hardware correlators is proposed. The previously adopted two-delay solutions were justified in the necessity of providing a phase reference to the correlator before the beginning of the correlation interval. However, this constraint can be relaxed by utilizing other phase reference and compensating the resulting correlation value accordingly after its computation. The proposed one-delay loop implementation is particularly useful for high dynamics conditions and therefore the UFA-PLL structure associated with an optimal digital loop filter was adopted for the analysis presented in this work. The optimum digital loop filter structures, previously derived for the case of two-delay carrier tracking loops, are updated under the one-delay condition. The ob-

Emails: agustinr@ing.unlp.edu.ar tained results are quite similar, with the novelty that the extra pole present in the former solution is not present any more. Two particular cases are analyzed by simulation to determine the pull-out performance and the associated tracking thresholds. First, a loop with correlation time of $T=10 \mathrm{~ms}$ is shown to be able to track acceleration steps of $20 \mathrm{~g}$ with similar performance than the previously obtained using two-delay implementations with $T=5 \mathrm{~ms}$ and the same $75.6 \mathrm{~Hz}$ bandwidth. In this case, doubling the correlation time imply a great reduction in the computational burden. Secondly, the same pull-out performance is shown in two $T=10 \mathrm{~ms}$ loops, one with two delays and $37.8 \mathrm{~Hz}$ bandwidth and the other with one delay and $19.8 \mathrm{~Hz}$ bandwidth. In this case, half bandwidth imply half variance of the estimated phase, which is equivalent to an improvement of $3 \mathrm{~dB}$ in the signal to noise ratio.

\section{INTRODUCTION}

Close-loop estimation schemes are usually chosen for realtime GNSS receivers due to their high computational efficiency. However, the presence of high user dynamics compromises the validity of well-known and long-established traditional carrier tracking techniques, thus calling for the development of new robust carrier tracking algorithms [1]. In [2] we proposed the Unambiguous Frequency Aided (UFA) PLL, a completely digital approach for these receivers, which has notable improvements over the classical and widely adopted FLL-assisted-PLL [3, 4]. The limitations in the analog-like loop design approach utilized up to that moment explain that the practical acceleration limit reported for GNSS tracking loop was $5 \mathrm{~g}$ and other more complex estimation structures were considered for high dynamics applications [5]. The pull-out performance of the UFAPLL was presented afterwards in [6], along with an analytical approximation that allows tuning the loop bandwidth and the correlation interval duration to minimize that probability. In that work, we showed that our UFA-PLL design optimized for accelerations steps of $20 \mathrm{~g}$ can produce a $3 \mathrm{~dB}$ 
improvement in tracking threshold over previous design approaches and that even for that accelerations the correlation interval does not need to be shorter than $5 \mathrm{~ms}$. The tracking threshold estimated with this approximation at that moment was $34 \mathrm{~dB} / \mathrm{Hz}$ for a loop with $75.6 \mathrm{~Hz}$ bandwidth. Actually, the tracking threshold of this loop was obtained more precisely by mean of Monte Carlo simulations presented in [7], where the value for $20 \mathrm{~g}$ acceleration steps is $31.5 \mathrm{~dB} / \mathrm{Hz}$. It is important to notice that the criterion utilized to define the tracking threshold in this later paper was modified from the one utilized in the former, considering how this threshold is measured experimentally.

One important aspect for the optimization of the loop filter is the number of delays present in the loop implementation. When the correlation stage is implemented in hardware, usually the correlation parameters have to be set before the starting time of the correlation interval. This fact imposes two hardware delays when closing the loop, situation that is typically present in GNSS receiver despite that sometimes it is not considered in the loop design. In [2] a digital loop design for carrier tracking loop was presented taking into account this constraint. Due to the presence of these two delays in the loop, the phase estimate obtained at a given instant is not computed with measurements up to this instant, but with measurements up to two previous instants. The real-time constraint does not allow taking advantage of this for the loop itself, but it is possible to improve its estimates by smoothing them with more recent measurements for other purposes as data detection and raw data generation for the navigation stage of GNSS receivers. Based on the loop state variables of the digital UFA-PLL design, optimally smoothed estimators for the phase and frequency of the received signals can be easily built [8].

In this work we present a new way of processing the correlations obtained in a real-time GNSS receiver with hardware correlators that allows implementing phase tracking loops with only one delay. The key idea behind this apparently contradictory statement is to realize that the phase utilized as reference by the correlator is not so relevant. Actually, after the result of the correlation has already been obtained it can be compensated so that the result is completely equivalent to the one obtained with another phase reference. By implementing this compensation, the annoying extra delay present in this kind of tracking loop implementations can be effectively avoided. That is not the case for the frequency estimate, which can affect the signal to noise ratio of the received signal if its error grows to levels comparable to the inverse of the correlation interval. Fortunately, the typical frequency errors obtained with either one-delay or two-delay optimum digital UFA-PLL loops implementations are at least one order of magnitude smaller, and therefore the compensation does not affect the behavior of the loop in terms of signal to noise ratio. Moreover, since the one-delay loop has a faster response, the ability to deal with high dynamics is improved without increasing the loop bandwidth, and without degrading the tracking threshold.

The paper is organized as follows. Section II review the discrete models used for the two-delay loos and justifies the possibility of implementing a real-time carrier tracking loop with only one-delay. The necessity of a frequency estimate in both cases, that can not be implemented with one delay is also discussed. Frequency estimates that can be derived from the same phase loop filter variables are proposed for both loop types. Simulations showing that the obtained frequency errors during acceleration steps are small enough are also presented. In section III we adapt the derivation of the optimum loop filter for acceleration steps presented in [2] for the one-delay loop implementation. The obtained results are coincident with the expressions for the optimal one step smoothed phase estimated proposed in [8] as a mean to improve the phase estimation error obtained with a two-delay loop. Notice that at that time the possibility of actually implementing a real-time carrier tracking loop with one-delay was not already conceived. Expressions and curves for obtaning the normalized loop bandwidth $B_{N} T$ are also provided. In section IV, the pull-out performance of different loop implementations are obtained by Monte Carlo simulation. Results show that tracking of $20 \mathrm{~g}$ acceleration steps can be effectively done with a $10 \mathrm{~ms}$ correlation interval tracking loop, which imply a great reduction in the computational burden in a GNSS receiver. The resulting tracking threshold is of approximately $32 \mathrm{~dB} / \mathrm{Hz}$ when the bandwidth is kept at $75.6 \mathrm{~Hz}$, the same value of the previous $5 \mathrm{~ms}$ two-delay implementation. Another example comparing two $10 \mathrm{~ms}$ loops is also presented, showing that the same pull-out performance can be obtained with half the bandwidth if the two-delay is replaced by a one-delay implementation. Finally, the conclusions are given in section $\mathrm{V}$.

\section{DISCRETE LOOPS MODEL}

The usual starting point for the model of a GNSS receiver tracking loop are the in-phase and in-quadrature correlations of the received signal with the locally generated replicas. For a given satellite received with carrier power to noise power spectral density $C / N_{0}$ and for the $i$-th integration interval of duration $T$, they can be expressed as [9]

$$
\begin{gathered}
I_{i}=D_{i} \operatorname{sinc}\left(\Delta f_{i}\right) R\left(\Delta \tau_{i}\right) \cos \left(\pi \Delta f_{i}+\Delta \theta_{i}\right)+n_{I i} \\
Q_{i}=D_{i} \operatorname{sinc}\left(\Delta f_{i}\right) R\left(\Delta \tau_{i}\right) \sin \left(\pi \Delta f_{i}+\Delta \theta_{i}\right)+n_{Q i}
\end{gathered}
$$

where $\Delta \tau_{i}=\tau_{i}-\hat{\tau}_{i}$ is the code delay estimation error, $\Delta f_{i}=$ $f_{i}-\hat{f}_{i}$ the frequency estimation error and $\Delta \theta_{i}=\theta_{i}-\hat{\theta}_{i}$ the phase estimation error, all assumed to be constant during the integration time. The sequences $n_{I i}$ and $n_{Q i}$ are uncorrelated white gaussian noise processes with variance 


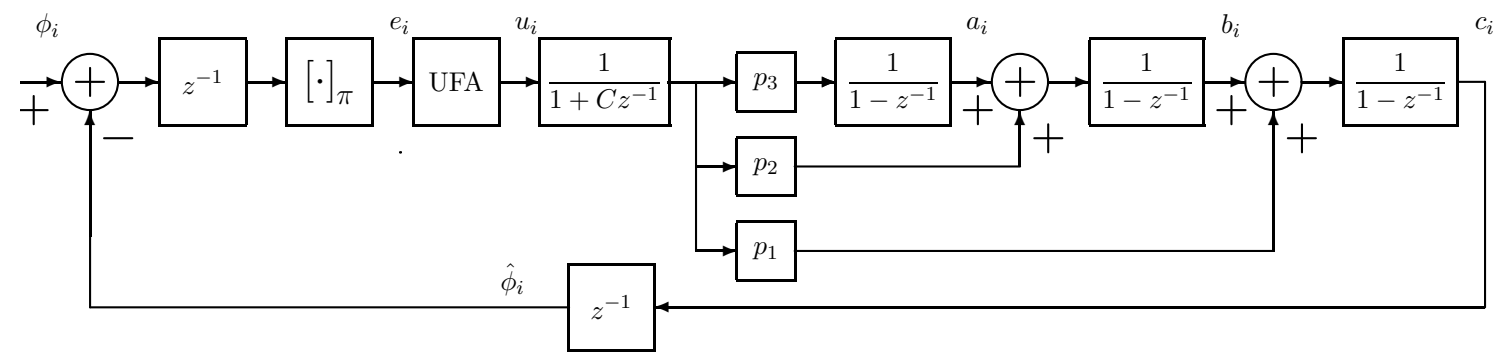

Figure 1. Block diagram of the UFA-PLL model with two delays.

$\sigma^{2}=1 /\left(2 T C / N_{0}\right), R(\cdot)$ is the code correlation function and $\operatorname{sinc}(x)=\sin (\pi x) /(\pi x)$. These expressions assume that there are binary data bits $D_{i}= \pm 1$ in the satellite signal, such as BPSK in the GPS civil signals or BOC modulation in modernized GNSS signals, and that the correlations are computed within one data-bit period. Thus, it is assumed that a bit synchronization stage has already taken place. After the initial code synchronization stage, small delay estimation errors can be assumed and then the function $R(\cdot)$ can be approximated by 1 , i.e. assuming perfect code synchronization. In this case, (1) and (2) reduce to

$$
\begin{gathered}
I_{i}=D_{i} \operatorname{sinc}\left(\Delta f_{i}\right) \cos \left(\Delta \phi_{i}\right)+n_{I i} \\
Q_{i}=D_{i} \operatorname{sinc}\left(\Delta f_{i}\right) \sin \left(\Delta \phi_{i}\right)+n_{Q i}
\end{gathered}
$$

where $\Delta \phi_{i}=\phi_{i}-\hat{\phi}_{i}$, with the definitions $\phi_{i}=\pi f_{i}+\theta_{i}$ and $\hat{\phi}_{i}=\pi \hat{f}_{i}+\hat{\theta}_{i}$, that can be interpreted as the input and estimated phases respectively corresponding to the middle of the correlation interval. The factor $\operatorname{sinc}\left(\Delta f_{i}\right)$ has been kept since it accounts for the effect of frequency errors that can be relevant in the tracking threshold determination although it is not considered directly in the carrier phase tracking loop model. A more convenient expression to handle the in-phase and in-quadrature correlations is to combine them into a complex one,

$$
C_{i}=I_{i}+j Q_{i}=D_{i} \operatorname{sinc}\left(\Delta f_{i}\right) e^{j \Delta \phi_{i}}+n_{i}
$$

where the sequence $n_{i}$ is a complex white Gaussian noise process with variance $\sigma_{c}^{2}=1 /\left(T C / N_{0}\right)$.

The carrier phase error, i.e. the phase of $C_{i}$, is typically obtained in digital loops using the inverse tangent phase discriminator because it is optimal (maximum likelihood estimator), it is not amplitude dependent, and the computational burden of calculating $\tan ^{-1}(\cdot)$ can be avoided with a short lookup table. Therefore,

$$
e_{i}=\tan ^{-1}\left(Q_{i} / I_{i}\right)=\angle C_{i}=\left[\Delta \phi_{i}+n_{\phi_{i}}\right]_{\pi} .
$$

The notation $[\cdot]_{\pi}$ indicates that its argument is kept within the interval $\left(-\frac{\pi}{2}, \frac{\pi}{2}\right]$ by adding or subtracting $\pi$ as many times as is needed. The zero-mean noise term $n_{\phi i}$ has a rather complicated probability distribution because of this non-linear transformation but in high $C / N_{0}$ conditions can be approximated by a gaussian distribution with zero mean and variance $\sigma^{2}$. A four-quadrant $\tan ^{-1}(\cdot)$ can only be used when tracking pilot signals, i.e. without data modulation. For the rest of GNSS signals, two quadrant versions are used since the discriminator becomes sensitive to the BPSK data modulation.

Regarding to the Unambiguous Frequency Aided (UFA) phase discriminator, it can be said that it adds memory to the previous phase discriminator in such a way that the implicit loop frequency error is taken into account and therefore it gives to the PLL the advantages of an FLL, but avoiding the annoying aspects of coupling two different loops in the so called FLL-asisted-PLL [3]. More concretely, in its two quadrant version the UFA phase discriminator corrects the instantaneous and ambiguous values of $e_{i}$ by adding or subtracting an integer number of $\pi$. This correction is such that the difference of successive values of the corrected phase error $u_{i}$ is less than half a cycle. The equation that implements the UFA phase error discriminator, with starting value $u_{0}=e_{0}$, is

$$
u_{i}=e_{i}-I_{\pi}\left(e_{i}-u_{i-1}\right)
$$

where $I_{\pi}(x)=x-[x]_{\pi}$ is an operation similar to the integer part function, but with steps at the multiples of $\pi$ [2]. One equivalent description of the UFA is to consider it as a modified $\tan ^{-1}(\cdot)$ function that produces output values in the range $\left(-\pi / 2+u_{i-1}, \pi / 2+u_{i-1}\right]$ instead of $(-\pi / 2, \pi / 2]$.

\subsection{Two delay UFA-PLL Model}

The digital loop model we were using during the last years, is shown in Fig. 1, where the filter coefficients $\left(p_{1}, p_{2}, p_{3}\right.$, and the extra pole in $z=C$ ) are defined by the loop filter design $[2,8]$. Since the loop filter is intended to track acceleration steps, it has three accumulators, i.e. a type 3 loop, plus an extra-pole that acts as a compensation for the two delays. It must be remembered that the two delays are present because of the real-time implementation of the loop feedback. One delay is due to the time spent in the $I$ and $Q$ calculations. The other delay appears because the estimated values used in the present correlations have to be known before the calculations begin. That is, the value 


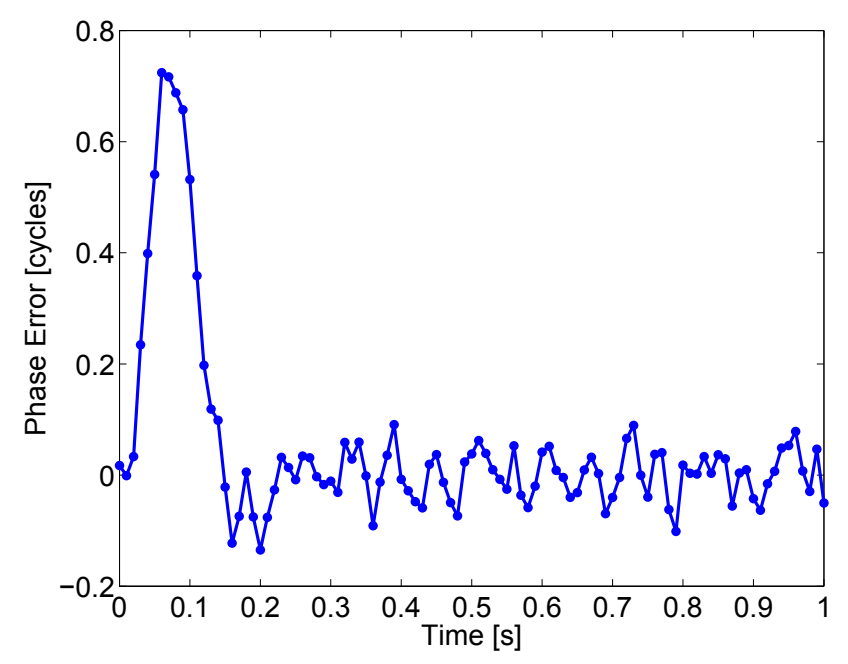

Figure 2. Phase error of two-delay UFA-PLL at $40 \mathrm{~g}$ step.

$\hat{\phi}_{i}$ is obtained with the loop filter output of the $(i-1)$-th correlation interval, which in turn is calculated with the estimation errors of $\hat{\phi}_{i-2}$. Hence, this second delay appears if the phase reference used for the correlation computation typically implemented in hardware- has to be provided by the loop output.

It is important to notice that also a frequency estimate is needed to generate the local replica for the correlations. The Single-Input Single-Output (SISO) models of Fig. 1 does not show explicitly which frequency estimate is used in the correlation. Since the output of the last accumulator $c_{i}$ is the loop phase estimate for the next correlation interval, $\hat{\phi}_{i+1}$, the output of the other two accumulators $b_{i}$ and $a_{i}$ can be interpreted as the loop estimates of frequency and change of frequency for the same interval respectively. However, as it was already mentioned, the loop tracks the sequence of phases at the middle of each correlation interval and $b_{i}$ is the difference between them. Hence, $b_{i}$ is a better frequency estimate for the beginning of the interval. Therefore, the frequency estimate for the $(i+1)$-th correlation interval can be improved doing

$$
\hat{f}_{i+1}=b_{i}+a_{i} / 2
$$

i.e., propagating the $b_{i}$ value to the middle of the interval with the loop change of frequency estimate. This frequency estimate can be shown to be unbiased when tracking acceleration steps. Also important is to consider the effect of amplitude reduction that the frequency error can produce in the received signal through the $\operatorname{sinc}(\cdot)$ function. As an example of the behavior of loops models that will be further characterized in the section IV, one snapshot of the phase and frequency errors of a two-delay UFA-PLL with $75.6 \mathrm{~Hz}$ bandwidth and $C / N_{0}=32 \mathrm{~dB} / \mathrm{Hz}$ are presented in Figs. 2 and. 3 for an acceleration step of $40 \mathrm{~g}$. In the latter it can be seen that -after the initial transient- the frequency

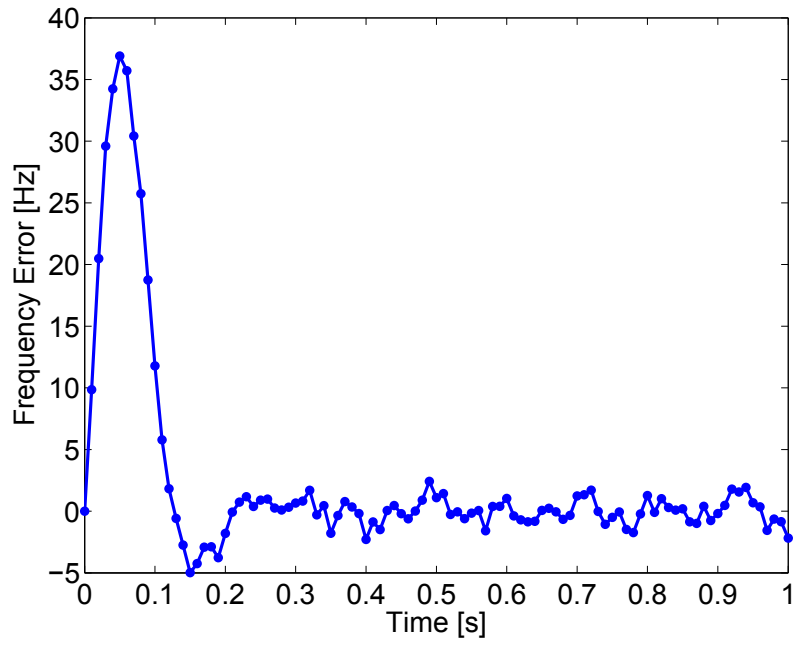

Figure 3. Frequency error of two-delay UFA-PLL at 40g step.

error is unbiased and that the maximum frequency error is about $37 \mathrm{~Hz}$. This error imply a signal level reduction of approximately $2 \mathrm{~dB}$ that can be of certain importance in the pull-out response of the loop.

\subsection{One delay UFA-PLL Model}

The key idea to implement a carrier tracking loop within a real-time GNSS receiver that has correlators implemented in hardware is to realize that the phase reference used for the correlation computation not necessarily has to be provided by the tracking loop. Indeed, other phase reference can be used and the obtained result will differ in a predictable way. Using the complex correlation expression (5), it is simple to notice that,

$$
\begin{aligned}
C_{i}=D_{i} \operatorname{sinc}\left(\Delta f_{i}\right) e^{j \Delta \phi_{i}}+n_{i}= \\
=\left(D_{i} \operatorname{sinc}\left(\Delta f_{i}\right) e^{j \phi_{i}}+n_{i}^{\prime}\right) e^{-j \hat{\phi}_{i}}
\end{aligned}
$$

where the sequence $n_{i}^{\prime}=n_{i} e^{j \hat{\phi}_{i}}$ is a statistically equivalent complex white Gaussian noise process like $n_{i}$. Therefore, the loop phase estimate can be applied after the correlation computation, i.e. rotating the correlation phase, without any side effect. The resulting scheme for such implementation is shown in Fig. 4, where for simplicity reasons the phase reference used in the hardware correlators was arbitrarily set to zero. The frequency estimate required for the local replica generation in the correlators requires more attention. In fact, it is actually the parameter that the loop needs to provide to the correlation hardware that can not be avoided. The advantage in this point is that, as long as the frequency estimation error is sufficiently small, the actual value of the frequency estimate is not relevant. In the new formulation of the loop, the output of the last accumulator $c_{i}$ is the loop phase estimate for the present correlation interval, $\hat{\phi}_{i}$, and the output of the other two accumulators 


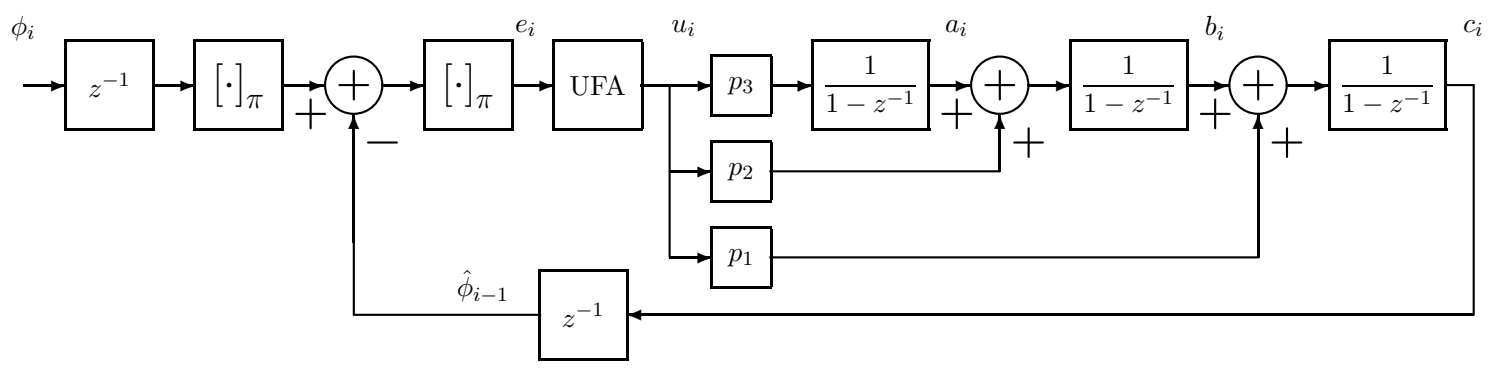

Figure 4. Block diagram of the UFA-PLL model with one delay.

$b_{i}$ and $a_{i}$ can still be used to build the frequency estimate. However, this value should be known one step in advance to be used in the hardware correlation. Then, the frequency estimate for the next correlation interval $\hat{f}_{i+1}$ has to be predicted with the values $b_{i}$ and $a_{i}$, which implies a propagation of the frequency estimate of one more sample time than before. Finally, an unbiased frequency estimate for the $(i+1)$-th correlation interval is

$$
\hat{f}_{i+1}=b_{i}+3 a_{i} / 2
$$

As it was done for the two-delay loop, one snapshot of the phase and frequency errors of a one-delay UFA-PLL with $75.6 \mathrm{~Hz}$ bandwidth and $C / N_{0}=32 \mathrm{~dB} / \mathrm{Hz}$ are presented in Figs. 5 and. 6 for an acceleration step of $40 \mathrm{~g}$. Comparing the former with Fig. 2, an important reduction in the phase error response can be appreciated, which anticipates a lower tracking threshold for this loop. In the case of the frequency error, it can be seen that effectively it is unbiased and that the maximum frequency error is a bit lower than before, approximately $28 \mathrm{~Hz}$. The corresponding signal level reduction is approximately $1 \mathrm{~dB}$, that will affect the correlations results during the peak transient response. Comparing with Fig. 3, it can also be noticed that after the transient the frequency error is somewhat higher than in the one-delay loop. As it was mentioned, for the purposes of the local replica generation there is no need of more refined estimator and therefore (10) was chosen because of its simplicity.

\section{LOOP FILTER DESIGN}

In this section, the digital loop filter design method presented in $[2,8]$ considering a two delay loop structure is reformulated accounting for the modification on the number of delays. The design of the digital loop filter is based on an optimization process that poses the typical loop bandwidth tradeoff in a quadratic functional which is minimized for a particular dynamic input. This criteria was proposed to obtain optimum analog loop filters in 1955 in [10], with results that became classics in PLL design literature. It consists in the minimization of a functional formed by the weighted sum of the output noise power of the loop $\sigma_{N}^{2}$, and the energy of the transient response of the loop error caused by a given input $\phi[n], E_{T}(\phi[n])$

$$
J=\sigma_{N}^{2}+\lambda^{2} E_{T}(\phi[n])
$$

where $\lambda^{2}$ is a weighting factor that controls the tradeoff between noise and transient response, i.e. the loop bandwidth. Since the functional uses the energy of the transient response, the optimum filter must produce a zero stationary response for the given input.

Calling $F(z)$ to the loop-filter transfer function to be found, without including the delay, and considering a linear model for the loop, its close loop transfer function is

$$
T(z)=\frac{F(z) z^{-1}}{1+F(z) z^{-1}}=Y(z) z^{-1}
$$

where $Y(z)$ can be any rational and stable transfer function. Then, the optimization process can be posed directly in terms of $Y(z)$, minimizing the functional $J(Y(z))$, in (11).

Assuming that the input noise is white with power espectral density $\eta / 2$, the variance of the output noise can be calculated as

$$
\sigma_{N}^{2}=\frac{\eta}{2} \int_{-\pi}^{\pi}\left|Y\left(e^{j \omega}\right)\right|^{2} \frac{d \omega}{2 \pi}=\frac{\eta}{j 4 \pi} \oint Y(z) Y\left(z^{-1}\right) \frac{d z}{z}
$$

where $Y\left(e^{j \omega}\right)$ is the frequency response of $Y(z)$ and the last integral extends over the unit circle of the complex plane.

Using Parseval theorem and naming $\phi_{e}[n]$ to the loop error response for the input $\phi[n]$, we can found

$$
\begin{array}{r}
E_{T}(\phi[n])=\sum_{n=0}^{\infty} \phi_{e}[n]^{2}=\int_{-\pi}^{\pi}\left|\phi_{e}\left(e^{j \omega}\right)\right|^{2} \frac{d \omega}{2 \pi}=\frac{1}{j 2 \pi} \\
\oint \phi(z) \phi\left(z^{-1}\right)\left[1-Y(z) z^{-1}\right]\left[1-Y\left(z^{-1}\right) z\right] \frac{d z}{z}
\end{array}
$$

where $\phi_{e}(z)=\phi(z)\left[1-Y(z) z^{-1}\right]$ is the $\mathrm{z}$ transform of $\phi_{e}[n]$ and $\phi(z)$ the $\mathrm{z}$ transform of $\phi[n]$. Then replacing (13) and (14) in (11)and defining $\gamma^{2}=2 \lambda^{2} / \eta$, we get the expression of the functional (11) with explicit dependence of $Y(z)$

$$
\begin{aligned}
J(Y(z))=\frac{\eta}{j 4 \pi} \oint & \left\{Y(z) Y\left(z^{-1}\right)+\gamma^{2} \phi(z) \phi\left(z^{-1}\right)\right. \\
& {\left.\left[1-Y(z) z^{-1}\right]\left[1-Y\left(z^{-1}\right) z\right]\right\} \frac{d z}{z} }
\end{aligned}
$$




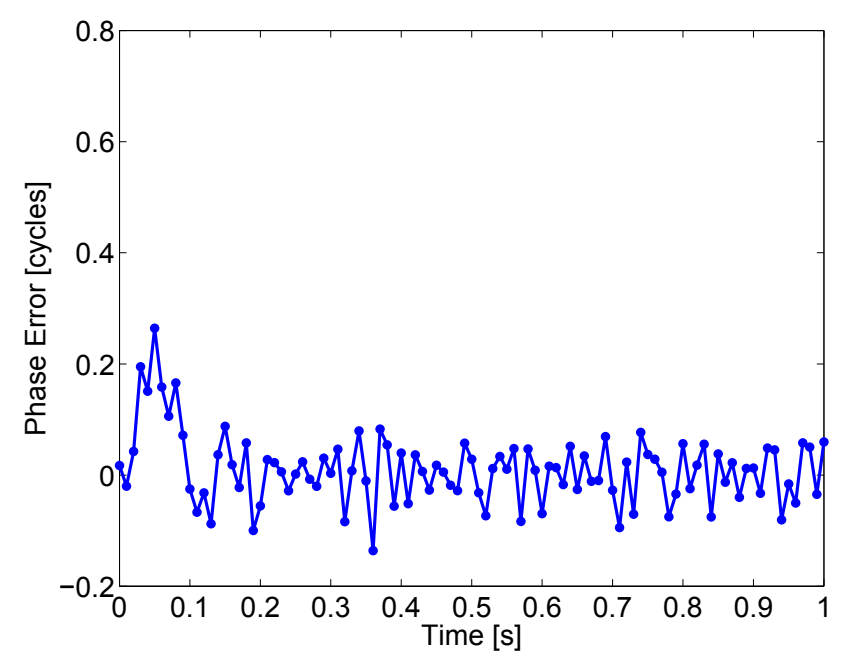

Figure 5. Phase error of a one-delay UFA-PLL at $40 \mathrm{~g}$ step.

which is minimized applying variational calculus.

The optimum $Y(z)$ obtained after these calculations is given by

$$
Y(z)=\frac{X(z) z}{\psi(z)}
$$

where $\psi(z)$ is a minimum phase rational function obtained from the following espectral decomposition

$$
\psi(z) \psi\left(z^{-1}\right)=1+\gamma^{2} \phi(z) \phi\left(z^{-1}\right) .
$$

This is always possible because the term on the right has an even number of poles and zeros. Poles, as well as zeros can be separated in two sets. If $z_{i}$ belongs to a set, $1 / z_{i}$ belongs to the other. Hnece, if $z_{i}$ is within the unit circle, there is also $1 / z_{i}$ outside the the unit circle. Finally a rational, minimum phase and stable $\psi(z)$ can be found. The function $X(z)$ is stable, rational and is found by the following parcial fractions decomposition

$$
\frac{\gamma^{2} \phi(z) \phi\left(z^{-1}\right)}{\psi\left(z^{-1}\right)}=X(z)+W\left(z^{-1}\right)
$$

That is, $X(z)$ retains the poles inside the unit circle and the rest form $W\left(z^{-1}\right)$, and then $W(z)$ is a stable function too.

It is interesting to note that only the last equation of the three that determine the loop filter is different from the two delays formulation. This relationship was exploited in [8] to create optimal smoothing structures based on the two delay loop filter estimates. Using these three equations, an optimum loop filter design can be obtained for a particular input that account for considered dynamic scenario. In particular, one case of interest practical is an acceleration step[2], and therefore the considered input is a frequency

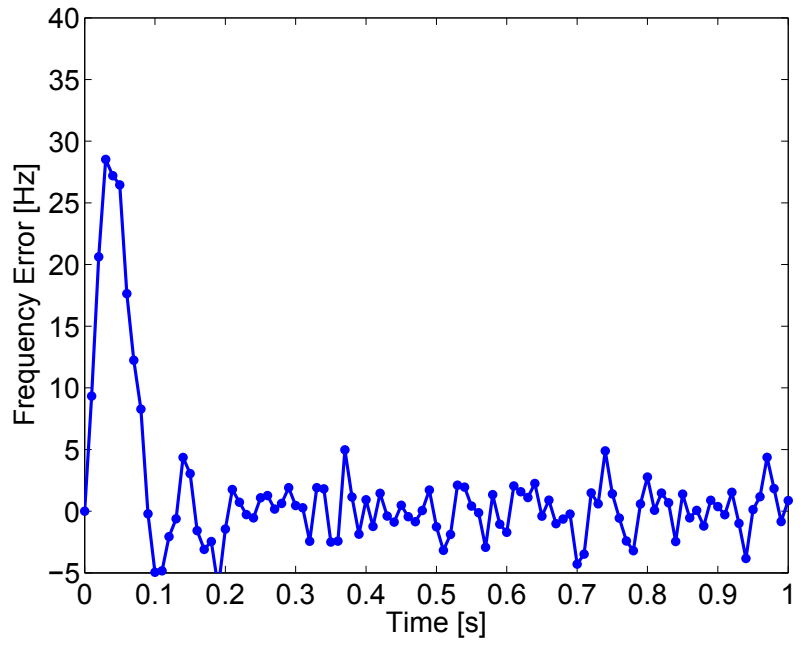

Figure 6. Frequency error of a one-delay UFA-PLL at $40 \mathrm{~g}$ step.

ramp

$$
\phi(z)=\frac{\dot{\Delta \omega} T^{2}}{\left(1-z^{-1}\right)^{3}}
$$

where $\Delta \omega$ is the rate of frequency change. Then, denoting $v=\Delta \omega^{2} T^{4} \gamma^{2},(z-1)^{6}-v z^{3}=0$ is the equation that determines the poles of the system. The six roots of this polynomial can be obtained using the fact that three of them are the inverses of the other three. This allows us to express the following equations

$$
\begin{aligned}
z_{1,2}+z_{1,2}^{-1} & =2-\frac{1 \pm j \sqrt{3}}{2} \sqrt[3]{v} \\
z_{3}+z_{3}^{-1} & =2+\sqrt[3]{v}
\end{aligned}
$$

that determine the values of $z_{1}$ and $z_{2}$ that are complex conjugates and $z_{3}$ real. Using these values and (17) it is found that

$$
\psi(z)=\frac{\left(1-z_{1} z^{-1}\right)\left(1-z_{2} z^{-1}\right)\left(1-z_{3} z^{-1}\right)}{\left(1-z^{-1}\right)^{3} z_{1}^{1 / 2} z_{2}^{1 / 2} z_{3}^{1 / 2}} .
$$

Finally, using Eq. (18) and Eq. (16) the optimum closed loop transfer function is

$$
Y(z)=\frac{C-(A-3 C) z^{-1}+(3 C-B) z^{-2}}{\left(1-z_{1} z^{-1}\right)\left(1-z_{2} z^{-1}\right)\left(1-z_{3} z^{-1}\right)}
$$

where $A=\left(6-3 z_{s}+z_{d}\right), B=\left(8-3 z_{s}+z_{p}\right)$ y $C=3-z_{s}$, with $z_{s}=z_{1}+z_{2}+z_{3}, z_{p}=z_{1} z_{2} z_{3}$ y $z_{d}=z_{1} z_{2}+z_{1} z_{3}+z_{2} z_{3}$.

And the optimum loop filter is

$$
F(z)=\frac{C-(A-3 C) z^{-1}+(3 C-B) z^{-2}}{\left(1-z^{-1}\right)^{3}} .
$$

Notice that the definition of the constants $A, B$ and $C$ has been kept equal to the ones stated in [2], and that the extra 


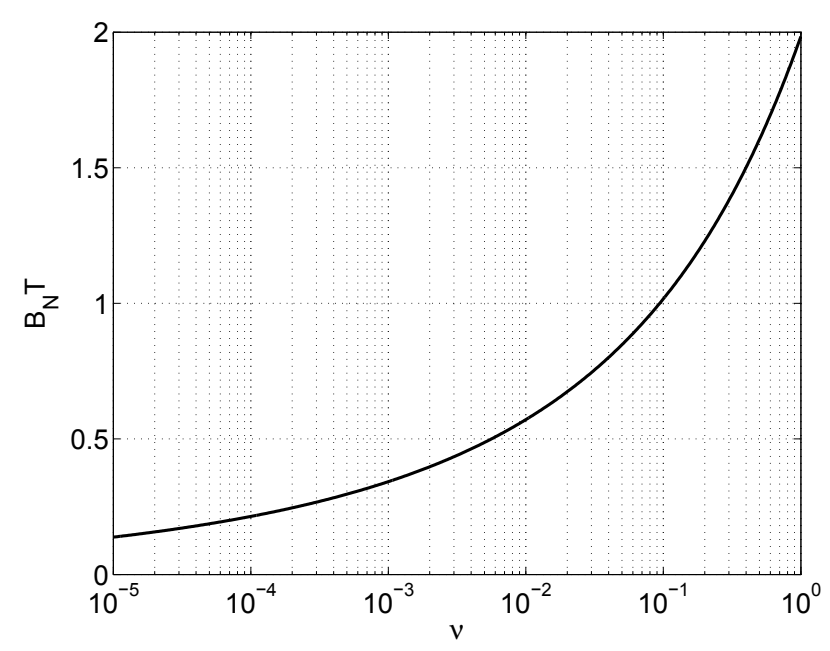

Figure 7. Normalized Noise Bandwidth and Peak Error Response for type 3 PLL

pole present in the two delay solution is not present any more. For the purpose of implementation, it is desirable to express the open loop transfer function as a cascade of accumulators. Then, (23) can be re-written as

$$
F(z)=\frac{p_{3}+p_{2}\left(1-z^{-1}\right)+p_{1}\left(1-z^{-1}\right)^{2}}{\left(1-z^{-1}\right)^{3}\left(1+p_{1} z^{-1}\right)}
$$

with $p_{1}=3 C-B, p_{2}=-A+2 B-3 C$, and $p_{3}=A-B+C$.

As it was done in previous cases, the closed loop equivalent noise bandwidth can be expressed as

$$
\begin{aligned}
B_{N} T=\Re e\left\{\operatorname{Res}_{z=z_{1}}\left\{Y(z) Y\left(z^{-1}\right)\right\}\right\} & \\
& +\frac{1}{2} \operatorname{Res}_{z=z_{3}}\left\{Y(z) Y\left(z^{-1}\right)\right\}
\end{aligned}
$$

and its plot is shown in Fig. 7, where the range of the parameter $v$ has been selected considering bandwidths of practical interest. Remember that values higher that one imply that the digital loop is actually amplifying the noise variance of the input phase noise.

\section{MONTE CARLO SIMULATIONS}

In this section, simulation to determine the performance a particular carrier tracking loop in terms of pull-out probability (POP) for different acceleration and signal levels are presented. Since a pull-out is an event that can be recognized in an advanced state, a frequency error criterion was selected to determine it in a relative early stage, as it was proposed in [7]. Due to the amplitude attenuation caused by the $\operatorname{sinc}(\cdot)$ function included in the simulations, a frequency error is equivalent to a signal power reduction. The adopted criterion was to declare pull-out if the frequency

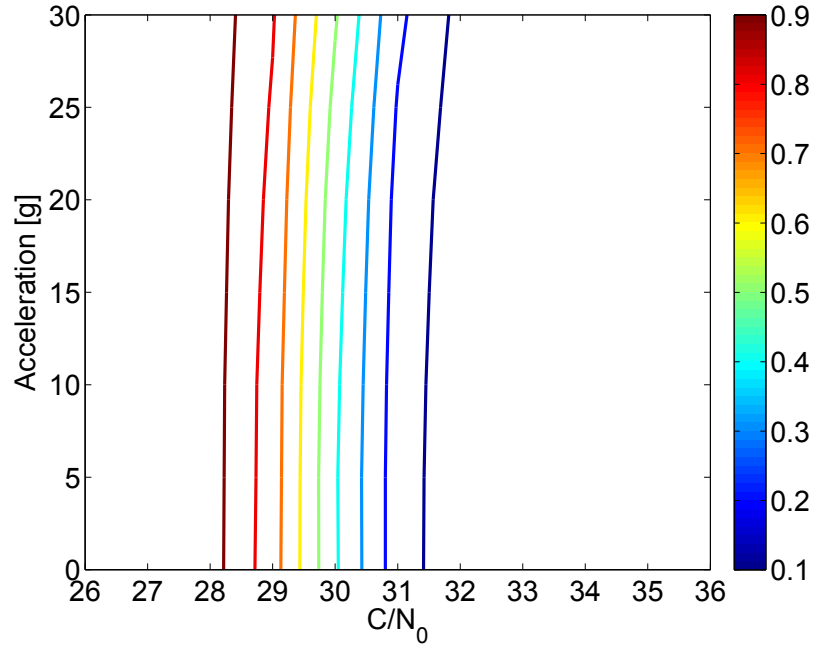

Figure 8. POP of Two-Delay UFA-PLL with $T=5 \mathrm{~ms}$ and $B w=75.6 \mathrm{~Hz}$

error exceeds $1 / T \mathrm{~Hz}$. In this situation, the signal power is completely attenuated and then the situation can be considered as an irrecoverable state. An error of less that $1 / T$ $\mathrm{Hz}$ is a critical situation but still recoverable. For each value of acceleration and $C / N_{0} 100.000$ runs of 1 second (100 or 200 samples depending the case) were computed. Using the previously mentioned criterion, which run presented a pull-out event was decided and then the POP estimated. Since the POP is computed for 1 second of tracking it can be also interpreted as the inverse of the mean-time to lose lock (MTLL) in seconds. Furthermore, the tracking threshold can be readily obtained based on this results since a practical way of defining it is as the $C / N_{0}$ that produce a POP of 0.1 in a period of one second.

In first term, the performance of the two-delay loop using $T=5 \mathrm{~ms}$ and bandwidth of $B_{N}=75.6 \mathrm{~Hz}$-proposed in [6] as an optimal design for tracking $20 \mathrm{~g}$ steps- is presented in Fig. 8 with results coincident with the analysis done in [7]. The resulting tracking threshold is slightly below $32 \mathrm{~dB} / \mathrm{Hz}$ for the whole range of accelerations simulated. In second term, the performance of a similar two delay loop with the same bandwidth but $T=10 \mathrm{~ms}$ in presented in Fig. 9 . Clearly, its performance -which is better in low dynamicsis severely affected by acceleration steps of $20 \mathrm{~g}$ and the tracking threshold becomes higher than $36 \mathrm{~dB} / \mathrm{Hz}$. This situation can be better addressed with a loop with the same bandwidth but with only one delay, which has the performance shown in Fig. 10. The obtained tracking threshold at $20 \mathrm{~g}$ is approximately $32 \mathrm{~dB} / \mathrm{Hz}$ and therefore almost the same performance of the $5 \mathrm{~ms}$ loop can be now obtained with a $10 \mathrm{~ms}$ loop, which imply a great reduction in the computational burden of the GNSS receiver. This behavior can be understood if it is noticed that two delays of 5 $\mathrm{ms}$ are equal to one delay of $10 \mathrm{~ms}$ and then both loops 


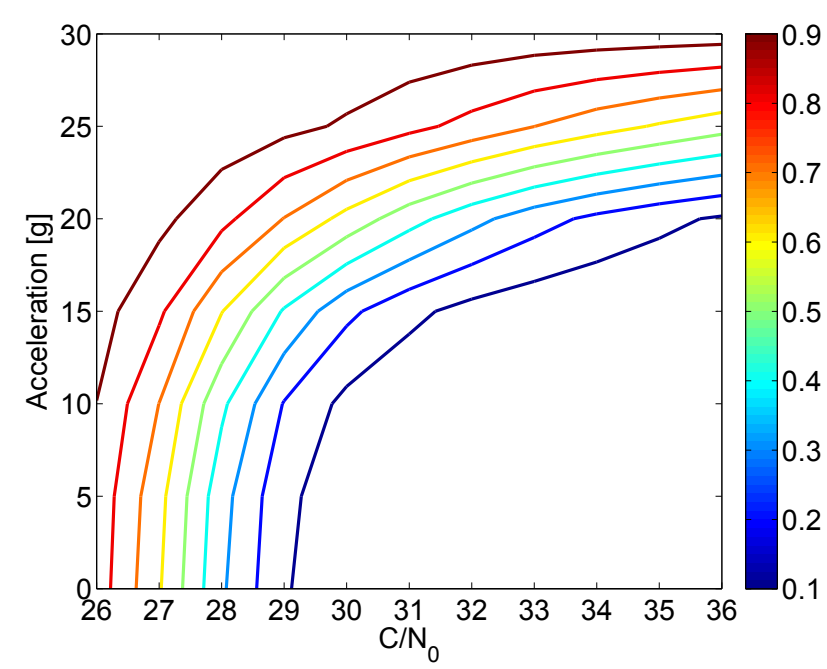

Figure 9. POP of Two-Delay UFA-PLL with $T=10 \mathrm{~ms}$ and $\mathrm{BW}=75.6 \mathrm{~Hz}$

implement an estimation structure with the same delay, but being the latter more efficient from the point of view of the implementation.

One more comparison using $T=10 \mathrm{~ms}$ is presented to show the advantages of implementing carrier tracking loops with only one delay. In Fig. 11 the performance of a two-delay loop with bandwidth $B_{N}=37.8 \mathrm{~Hz}$ (half of the previous one) is presented. As expected, its behavior its very good in low dynamics but unacceptable for accelerations higher that $10 \mathrm{~g}$. Then, in Fig. 12 the performance of a one-delay loop with bandwidth $B_{N}=18.9 \mathrm{~Hz}$ (a quarter of the previous one) which is practically the same in the whole simulated range of accelerations and signals to noise ratios. Hence, using the one-delay loop the phase estimation variance can be reduced by a half without any additional computational complexity and without any reduction in the pullout resistance of the implemented carrier tracking loop.

\section{CONCLUSIONS}

The possibility of implementing one-delay carrier tracking loops in GNSS receivers that utilized hardware correlators has been clearly stated. The constraint of providing a phase reference to the correlator before the beginning of the correlation interval can be simply relaxed by utilizing other reference value -zero for instance- and compensating the resulting correlation value accordingly after its computation. The local replica frequency still needs to be provided before the computation starts, but in this case good enough two-delay frequency predictions can be obtained based on the loop filter variables. As long as the frequency error is sufficiently small compared with the inverse of the correlation interval duration, $T$, the amplitude reduction effect caused can be neglected and the closed loop phase estima-

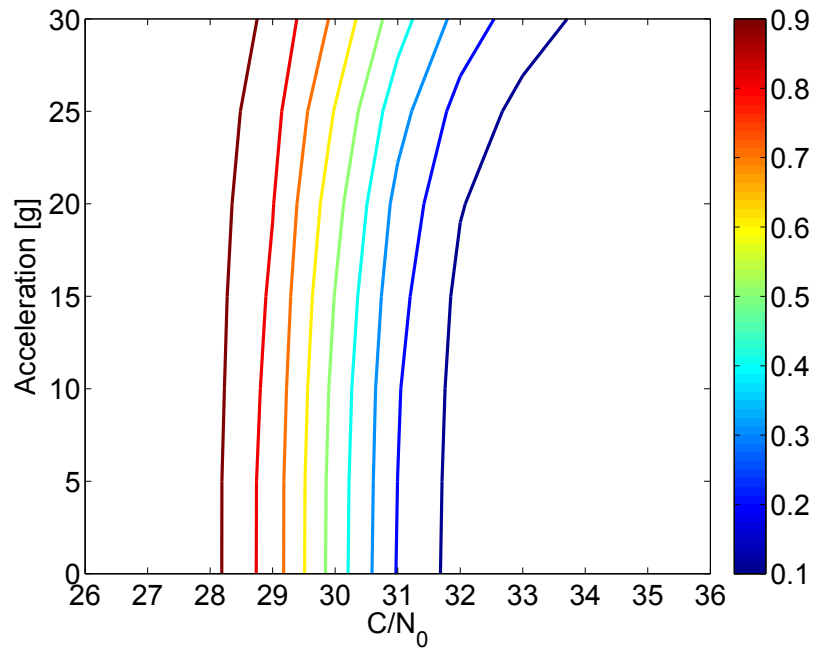

Figure 10. POP of One-Delay UFA-PLL with $T=10$ and $B w=75.6 \mathrm{~Hz}$

tion process properly done. The optimum digital loop filter structure, for a generic input dynamics and in particular for acceleration steps was derived, as it was previously done for the case of two-delay carrier tracking loops. The obtained results are quite similar, with the novelty that the extra pole present in the former solution is not present any more.

The presence of delays in a digital loop implementation is crucial when it is intended for operation in high dynamics conditions. In that cases, the UFA-PLL structure associated with an optimal digital loop filter design outperforms other previously proposed closed loop structures, and therefore it was the selected option for the analysis presented in this work. The application of the proposed strategy to reduce the delays present in a carrier loop implementation for loops intended for operation in low dynamics scenarios, or for carrier-aided code tracking loops, is of minor importance due to fact that the utilized bandwidth are so small that even a continuos-time model of the loop is acceptable.

The convenience of implementing the one-delay carrier tracking loop scheme was determined by simulation. In first place, it was shown that acceleration steps of $20 \mathrm{~g}$ can be properly tracked using correlation times of $T=10 \mathrm{~ms}$. The performance of the loop is similar to the previously obtained using two-delay implementations with $T=5 \mathrm{~ms}$ and the same $75.6 \mathrm{~Hz}$ bandwidth. It should be emphasized that doubling the correlation time imply a great reduction in the computational burden required for the operation of the carrier tracking loop in a real-time GNSS receiver. In second place, it was shown that in a $T=10 \mathrm{~ms}$ loop implementation, the change from two to one delay allows to reduce the loop bandwidth to half of the original value maintaining the pull-out performance of the loop unaltered. In this case, the use of a one-delay loop allows to reduce the vari- 


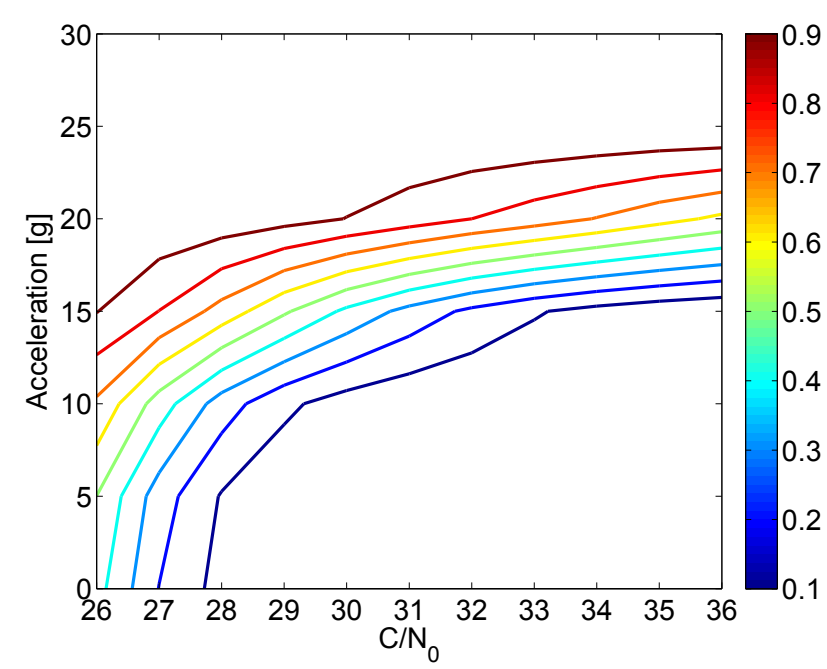

Figure 11. POP of Two-Delay UFA-PLL with $T=10 \mathrm{~ms}$ and $B w=37.8 \mathrm{~Hz}$

ance of the estimated phase to the half, which is equivalent to an improvement of $3 \mathrm{~dB}$ in the signal to noise ratio.

\section{ACKNOWLEDGEMENT}

This work was supported by National Agency for Promotion of Science and Technology (ANPCyT), the National Research Council (CONICET), the Buenos Aires Province Research Council (CIC-PBA) and the National University of La Plata (UNLP), all from Argentina.

\section{REFERENCES}

[1] J. A. López-Salcedo, J. A. Del Peral-Rosado, and G. Seco-Granados, "Survey on robust carrier tracking techniques," IEEE Communications Surveys and Tutorials, vol. 16, no. 2, pp. 670-688, 2014.

[2] P. A. Roncagliolo and J. G. García, "High Dynamics and False Lock Resistant GNSS Carrier Tracking Loops," in Proceedings of The 20th International Technical Meeting of The Satellite Division of The Institute of Navigation, ION GNSS 2007, Fort Worth, Texas, September 2007.

[3] E. D. Kaplan, Understanding GPS: Principles and Applications. Boston: Artech House, 1996.

[4] P. W. Ward, "Performance comparisons between FLL, PLL and a novel FLL-assisted-PLL carrier tracking loop under RF interference conditions," in Proceedings of The 11th International Technical Meeting of The Satellite Division of The Institute of Navigation, ION GPS 1998, Nashville, Tennessee, September 1998 .

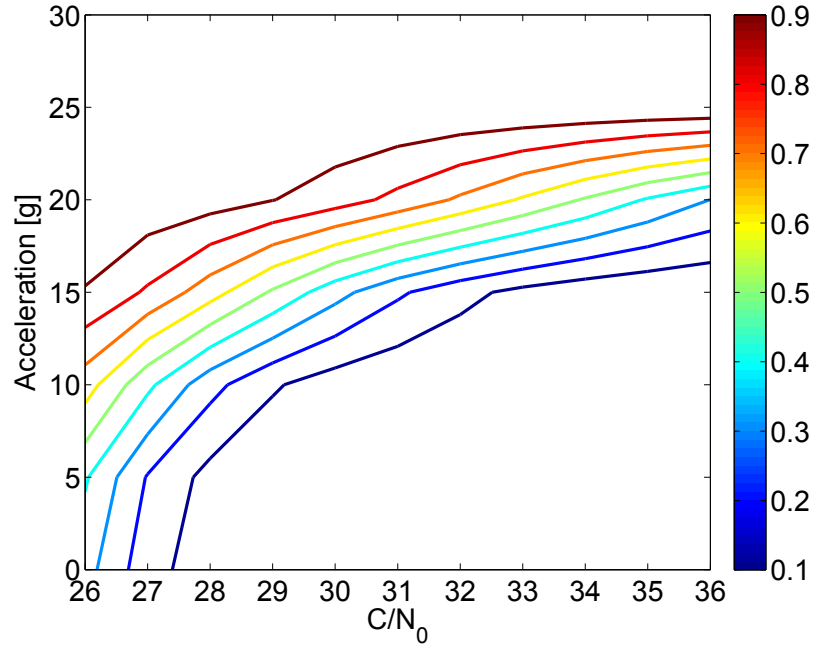

Figure 12. POP of One-Delay UFA-PLL with $T=10 \mathrm{~ms}$ and $B w=18.9 \mathrm{~Hz}$

[5] W. J. Hurd, J .I. Statman, and V. A. Vilnrotter, "High dynamic gps receiver using maximun likelihood estimation and frequency tracking," IEEE Transactions on Aerospace and Electronic Systems, vol. 23, no. 4, pp. 425-437, July 1968.

[6] P. A. Roncagliolo, J. G. García and C.H. Muravchik, "Pull-out Probability and Tracking Threshold Analysis for High Dynamics GNSS Carrier Loops," in Proceedings of The 21th International Technical Meeting of The Satellite Division of The Institute of Navigation, ION GNSS 2008, Savannah, Georgia, September 2008.

[7] _ _ "A Joint Carrier and Data Estimation Scheme for Real-Time High Dynamics GNSS Receivers," in Proceedings of The 5th Advanced Satellite Multimedia Systems Conference and the 11th Signal Processing for Space Communications Workshop, ASMSSPSC 2010, Cagliari, Italy, September 2010.

[8] — - "Optimized carrier tracking loop design for real-time high-dynamics gnss receivers," International Journal of Navigation and Observation, vol. 2012, p. 18, July 2012.

[9] B. W. Parkinson and J. J. Spilker (eds.), Global Positioning System: Theory and Applications. Washington: American Institute of Aeronautics and Astronautics (AIAA), 1996.

[10] R. Jaffe and E. Rechtin, "Design and performance of phase-lock circuits capable of near-optimun performance over a wide range of inputs signal and noise levels," IEEE Transctions on Information Theory, vol. 1, no. 1, pp. 66-76, 1955. 